\title{
Effective diffusivity of passive scalars in rotating turbulence
}

\author{
P. Rodriguez Imazio $^{1}$ and P. D. Mininni ${ }^{1,2}$ \\ ${ }^{1}$ Departamento de Física, Facultad de Ciencias Exactas y Naturales, Universidad de Buenos Aires and IFIBA, CONICET, \\ Cuidad Universitaria, Buenos Aires 1428, Argentina \\ ${ }^{2}$ National Center for Atmospheric Research, P.O. Box 3000, Boulder, Colorado 80307, USA
}

(Received 17 October 2012; published 25 February 2013)

\begin{abstract}
We use direct numerical simulations to compute turbulent transport coefficients for passive scalars in turbulent rotating flows. Effective diffusion coefficients in the directions parallel and perpendicular to the rotation axis are obtained by studying the diffusion of an imposed initial profile for the passive scalar, and calculated by measuring the scalar average concentration and average spatial flux as a function of time. The Rossby and Schmidt numbers are varied to quantify their effect on the effective diffusion. It is found that rotation reduces scalar diffusivity in the perpendicular direction. The perpendicular diffusion can be estimated from mixing length arguments using the characteristic velocities and lengths perpendicular to the rotation axis. Deviations are observed for small Schmidt numbers, for which turbulent transport decreases and molecular diffusion becomes more significant.
\end{abstract}

DOI: 10.1103/PhysRevE.87.023018

PACS number(s): 47.27.-i, 51.20.+d

\section{INTRODUCTION}

Turbulent transport in anisotropic flows plays an important role in a wide variety of astrophysical and geophysical processes (see, e.g., [1-3] for examples of transport in astrophysics, [4] for an example in the context of the geodynamo, [5] for an example in atmospheric flows, and [6] for a study of vertical transport and diffusion in the ocean). In many of these examples, the resulting mixing is often studied and modeled by means of anisotropic transport coefficients $[5,7,8]$.

While the transport of passive scalars in isotropic turbulent flows has received a lot of attention (see, e.g., [9-11]), fewer studies have considered the case of diffusion of the passive scalar in anisotropic flows. In the presence of anisotropy, the transport of the passive scalar is modified and turbulent transport coefficients should become anisotropic. Of particular relevance for the atmosphere and the oceans (as well as for mixing in stellar convective regions) are the cases or rotating and/or stratified turbulence. In the case of stratified flows, numerical simulations [7] showed that the stratification acts to reduce the horizontal fluctuations of a scalar (i.e., it maintains the turbulent mixing in horizontal planes), while it suppresses the vertical mixing and transport of the passive scalar. These results were also confirmed analytically later in [12].

Turbulent transport and diffusion of passive scalars in rotating flows has received less attention, although the anisotropy introduced by rotation is important at the largest scales of the atmosphere and the oceans, as well as in structures in the atmosphere that develop strong local rotation, as in tornadoes $[13,14]$. In the astrophysical context, the effects of rotation on turbulent diffusion are also important to understand the development of latitudinal entropy gradients in rotating stars [8]. Furthermore, anisotropic passive scalar transport is believed to be associated with the partial depletion of lithium observed in the Sun [3].

The development of anisotropy in rotating flows differs from that in stratified flows. While in the latter case structures tend to be flat and layered, in rotating flows structures are elongated along the axis of rotation and the flow becomes quasitwo-dimensional [15]. In rotating flows, turbulent transport coefficients for passive scalars were obtained from numerical simulations using the test field model in [8]. The results indicate that turbulent diffusion in the direction of rotation is reduced when the scales of the turbulence are comparable with those of the mean field. Recently, we considered scaling laws and intermittency of the passive scalar in rotating flows [16] and found that the distribution of the passive scalar becomes highly anisotropic, with scaling laws developing in the plane perpendicular to the rotation axis, and with scaling compatible with Kraichnan's model [17] for the passive scalar in two dimensions.

In a more general context, many authors have studied turbulent diffusion in anisotropic flows in terms of particle dispersion. These studies are related with the transport of passive scalars, as the average concentration of a passive scalar is related to single-particle dispersion in a fluid. Theoretical and numerical studies [18,19] (see also [20-22], and simulations in [23]) indicate one-particle dispersion is suppressed along the direction of stratification in stably stratified turbulence with and without rotation. Moreover, results in [19] and [22] show that the anisotropy introduced by stratification also reduces horizontal diffusion, although to a much lesser extent than vertical diffusion. These results are in good agreement with previous experimental results [24]. In the case of rotating flows without stratification, the same studies indicate that horizontal diffusion is highly reduced, while vertical diffusion remains nearly unchanged. More recently, Lagrangian velocity autocorrelations (related with turbulent diffusion) were obtained in experiments of forced rotating turbulence [25], and numerical simulations considered mixing in rotating flows [26].

In this work, we study turbulent transport coefficients for the passive scalar in a turbulent rotating flow. Following a procedure similar to the one used in [7] for stratified flows, effective coefficients are obtained by studying the diffusion of an initial distribution of the scalar quantity in a turbulent flow, and calculated by measuring the average concentration and average flux of the scalar. Unlike previous studies (see, e.g., [8]), the coefficients obtained here are not scale dependent. Coefficients calculated in this fashion are useful when a computationally economical description of the problem is required, as is often the case, e.g., in weather and climate modeling [13]. As small-scale turbulence in weather 
simulations can not be explicitly resolved (as an example, resolving small-scale turbulence in hurricanes would require

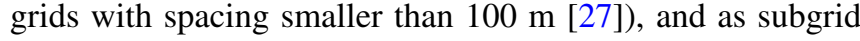
models such as large-eddy simulations require a fraction of the small-scale turbulence spectrum to be sufficiently resolved, a large fraction of numerical models in atmospheric sciences use simple turbulence parametrization schemes with effective diffusion and mixing coefficients [28]. These numerical models are known to produce unphysical results if turbulence is not properly parametrized (see, e.g., [29,30]). Determination of anisotropic transport coefficients is thus important for such numerical and theoretical models.

In our study, the velocity field and the passive scalar concentration are obtained from direct numerical simulations (DNS) of the Navier-Stokes equations in a rotating frame, together with the advection-diffusion equation for the scalar concentration. All DNS are performed using $512^{3}$ grid points in a regular periodic tridimensional grid. Three different rotation rates are considered, chosen to study turbulence with moderate Rossby number. Diffusion coefficients are calculated for the vertical (i.e., parallel to the rotation axis) and horizontal directions. A more detailed study of turbulent diffusion is then made varying the Schmidt number, in order to observe Schmidt and Pèclet number dependence. Finally, we present visualizations of the scalar which allow a more complete interpretation of the diffusive processes in the vertical and horizontal directions.

We find that rotation dramatically reduces scalar diffusivity in the horizontal direction (in comparison with the isotropic and homogeneous case). This is in good agreement with theoretical arguments in [22] for single-particle dispersion, where it was found that vertical diffusion is twice larger than horizontal diffusion in the presence of pure rotation. Within error bars, our results are consistent with this finding. Moreover, we find that horizontal diffusion can be reasonably estimated using mixing length arguments as $U_{\perp} L_{\perp}$ (where $U_{\perp}$ and $L_{\perp}$ are, respectively, the characteristic velocity and length scale in the direction perpendicular to the rotation axis). For small Schmidt and Pèclet numbers, the turbulent diffusion decreases as molecular diffusion becomes more important, while for large enough Pèclet numbers, the effective diffusion becomes independent of the Pèclet number.

\section{NUMERICAL SIMULATIONS}

\section{A. Equations and parameters}

Data analyzed in the following section stems from DNS of the incompressible Navier-Stokes equations in a rotating frame for the velocity $\mathbf{u}$, and of the advection-diffusion equation for the passive scalar $\theta$, given by

$$
\begin{gathered}
\partial_{t} \mathbf{u}+\mathbf{u} \cdot \nabla \mathbf{u}=-2 \Omega \times \mathbf{u}-\nabla p+\nu \nabla^{2} \mathbf{u}+\mathbf{f}, \\
\nabla \cdot \mathbf{u}=0, \\
\partial_{t} \theta+\mathbf{u} \cdot \nabla \theta=\kappa \nabla^{2} \theta .
\end{gathered}
$$

Here, $p$ is the pressure divided by the mass density (chosen uniform and constant in all simulations), $v$ is the kinematic viscosity, and $\kappa$ is the scalar diffusivity. Also, $\mathbf{f}$ is an external force that drives the turbulence, and $\Omega=\Omega \hat{z}$ where $\Omega$ is the rotation angular velocity. The mechanical forcing $\mathbf{f}$ is a superposition of Fourier modes with random phases, deltacorrelated in time, injected in a narrow band of wave numbers $k \in[1,2]$ (therefore, we will consider the forcing wave number as $\left.k_{F} \approx 1\right)$.

Equations (1), (2), and (3) are solved in a three-dimensional domain of size $2 \pi$ with periodic boundary conditions using a parallel pseudospectral code $[31,32]$. The pressure is obtained by taking the divergence of Eq. (1), using the incompressibility condition (2), and solving the resulting Poisson equation. The equations are evolved in time using a second-order RungeKutta method. The code uses the $\frac{2}{3}$ rule for dealiasing, and as a result the maximum wave number is $k_{\max }=N / 3$, where $N$ is the number of grid points in each direction (with $N=512$ in all the runs). All simulations presented are well resolved, in the sense that the dissipation wave numbers $k_{v}$ and $k_{\kappa}$ are smaller than the maximum wave number $k_{\max }$ at all times.

The dimensionless numbers used to characterize the runs are the Reynolds, Pèclet, and Rossby numbers, defined, respectively, as

$$
\begin{gathered}
R_{e}=\frac{U L}{v}, \\
P_{e}=S_{c} R_{e}=\frac{v}{\kappa} R_{e}, \\
R_{o}=\frac{U}{2 L \Omega},
\end{gathered}
$$

where $U$ is the root mean square (rms) velocity in the turbulent steady state, and $L$ is the forcing scale of the flow defined as $L=2 \pi / k_{F} . S_{c}$ is the Schmidt number, defined as $S_{c}=v / \kappa$. In all simulations, $U \approx 1$, and the viscosity is $v=6 \times 10^{-4}$. The passive scalar diffusivity $\kappa$ is set equal to the viscosity for the main set of runs, although similar simulations but changing the value of $S_{c}$ (and therefore of $P_{e}$ ) were also performed.

The detailed procedure followed in the numerical simulations is as follows. We first conducted a simulation solving Eqs. (1) and (2) (Navier-Stokes without passive scalar and without rotation), starting from the fluid at rest $(\mathbf{u}=0)$, and applying the external forcing $\mathbf{f}$ until reaching a turbulent steady state. This run was continued for approximately 13 turnover times. The final state of this run was used as the initial condition for the velocity field in multiple runs in which the external forcing $\mathbf{f}$ was kept the same but a passive scalar was injected. These runs can be grouped (namely, in sets $A, B, C$, and $E$ ), with each set corresponding to several runs with the same Rossby number (see Table I).

Each run in each set corresponds to a simulation in which an initial Gaussian profile for the passive scalar was injected

TABLE I. Parameters used in each set of runs. $\Omega$ is the rotation rate, $R_{o}$ is the Rossby number, $R_{e}$ is the Reynolds number, and $U$ is the rms velocity in the turbulent steady state.

\begin{tabular}{llccc}
\hline \hline Set & $\Omega$ & $R_{o}$ & $R_{e}$ & $U$ \\
\hline$A$ & 0 & $\infty$ & 1050 & 1 \\
$B$ & 2 & 0.04 & 1050 & 1 \\
$C$ & 4 & 0.02 & 1050 & 1 \\
$E$ & 8 & 0.01 & 1050 & 1 \\
\hline \hline
\end{tabular}


TABLE II. Parameters used in one of the subsets of runs. In the name of each run, the subindex $x$ or $z$ indicates the dependence with the coordinate of the initial passive scalar Gaussian profile, and the subindex with the number is $1 / S_{c}$. For each run, $v$ is the kinematic viscosity, $\kappa$ is the molecular diffusivity, $P_{e}$ is the Pèclet number, and $S_{c}$ is the Schmidt number.

\begin{tabular}{lcrrc}
\hline \hline Run & $v$ & $\kappa$ & $P_{e}$ & $S_{c}$ \\
\hline$A_{x 1 / 2}$ & $6 \times 10^{-4}$ & $3 \times 10^{-4}$ & 2100 & 2 \\
$A_{x 1}$ & $6 \times 10^{-4}$ & $6 \times 10^{-4}$ & 1050 & 1 \\
$A_{x 2}$ & $6 \times 10^{-4}$ & $1.2 \times 10^{-3}$ & 520 & 0.5 \\
$A_{x 4}$ & $6 \times 10^{-4}$ & $2.4 \times 10^{-3}$ & 260 & 0.25 \\
$A_{x 8}$ & $6 \times 10^{-4}$ & $4.8 \times 10^{-3}$ & 130 & 0.12 \\
$A_{x 16}$ & $6 \times 10^{-4}$ & $9.6 \times 10^{-3}$ & 66 & 0.06 \\
$A_{x 32}$ & $6 \times 10^{-4}$ & $1.92 \times 10^{-2}$ & 33 & 0.03 \\
\hline \hline
\end{tabular}

as follows:

$$
\theta\left(t=0, x_{i}\right)=\theta_{0} e^{-\left(x_{i}-\mu\right)^{2} / \sigma^{2}},
$$

where $i=1$ or 3 (i.e., the initial profile is a function of $x_{1}=x$ or $x_{3}=z$ ), $\mu=\pi$ (the profile is centered in the middle of the box, with the box of length $2 \pi$ ), and $\sigma=1$. This allows us to study the diffusion of the initial profile in the direction parallel to rotation $(z$, or vertical) and in the direction perpendicular $(x$, or horizontal). For a few runs, it was explicitly verified that the diffusion in the $x$ and $y$ directions was the same (as rotating turbulence tends to be axisymmetric). The runs in each set are labeled with a subindex indicating the dependence of the initial profile (e.g., runs in group $A$ are labeled $A_{x}$ or $A_{z}$ depending on the initial Gaussian profile used).

Finally, to measure the effect of varying the Schmidt number on the effective diffusivity, all simulations in each group (with subscript $x$ or $z$ ) were repeated with different values of $\kappa$. In practice, we performed simulations increasing the molecular diffusivity from $\kappa=v$ (all the simulations described above) to $\kappa=32 v$ (increasing $1 / S_{c}$ by factors of 2 for each run). Simulations with $\kappa=v / 2$ were also performed. This results in 12 more simulations in each group, with $S_{c}=2$ to $\frac{1}{32}$. To differentiate each run in each set, a subindex equal to $1 / S_{c}$ is added after the subindex $x$ or $z$. The resulting list of all runs in set $\mathrm{A}$ is shown in Table II, indicating the value of $v$, $\kappa$, and the corresponding $P_{e}$ and $S_{c}$ numbers. The runs in the other sets are labeled following the same rules, and have the same parameters as those shown in Table II. As an example, runs $A_{x 4}$ and $C_{x 4}$ have the same values of $\nu, \kappa, P_{e}$, and $S_{c}=\frac{1}{4}$, with an initial Gaussian profile for the passive scalar in $x$. The two runs differ just in the Rossby number.

Since for each set of values of $R_{0}$ we considered seven different Schmidt numbers, and since for each of these sets of parameters we performed two runs (one for the initial profile of the passive scalar in the $x$ direction, and one for the profile in the $z$ direction), the total number of numerical simulations analyzed below is 56 .

\section{B. Turbulent diffusion coefficients}

To characterize the turbulent diffusion of the passive scalar, we consider quantities averaged over the two directions perpendicular to the initial dependence of the Gaussian profile. In particular, we consider the average passive scalar concentration $\bar{\theta}$ and the spatial flux $\overline{\theta u_{i}}$, where $i=1$ or 3 depending on the initial dependence of the Gaussian profile, and the averages are done over the two remaining Cartesian coordinates. Note the spatial flux $\overline{\theta u_{i}}$ represents the amount of passive scalar transported in the $i$ direction per unit of time by the fluctuating (or turbulent) velocity. Since there is no mean flow in the simulations (we use delta-correlated in time random-forcing), $u_{i}$ is the fluctuating velocity. Then, the pointwise effective turbulent diffusion coefficient at $x_{i}$ and at a given time $t$ is [7]

$$
\mathcal{D}_{i}\left(x_{i}, t\right)=\frac{\overline{\theta u_{i}}}{\partial_{x_{i}} \bar{\theta}} .
$$

This coefficient corresponds to how much passive scalar is transported by the fluctuating velocity, per unit of variation of $\bar{\theta}$ with respect to $x_{i}$. As already mentioned, $i=1$ will stand for horizontal diffusion, while $i=3$ will stand for vertical diffusion.

In nature, turbulence often results from an instability of a large-scale flow (e.g., convection in stars, in the Earth's core and in the atmosphere, or the general circulation at the largest scales in the atmosphere and the oceans). The random forcing described above is intended to mimic the behavior of rotating turbulence at scales smaller than the large-scale flow; together with the rotation it produces an anisotropic flow with columnlike structures, but it does not produce a coherent mean flow. When such a mean flow is present, Eq. (8) remains valid but the fluctuating velocity has to be defined from the total velocity field $\mathbf{v}$ after subtracting the mean flow

$$
\mathbf{u}=\mathbf{v}-\langle\mathbf{v}\rangle,
$$

where the mean flow $\langle\mathbf{v}\rangle$ can be defined, e.g., from a time average, in such a way the mean value of the fluctuating velocity $\langle\mathbf{u}\rangle$ is zero.

From Eq. (8), we can define averaged diffusion coefficients as follows. We can first average over the coordinate $x_{i}$ to obtain a time dependent turbulent diffusion

$$
\mathcal{D}_{i}(t)=\frac{1}{2 \pi} \int_{0}^{2 \pi} \mathcal{D}_{i}\left(x_{i}, t\right) d x_{i},
$$

and we can further average over time to obtain the mean turbulent diffusion

$$
\mathcal{D}_{i}=\frac{1}{T} \int_{t_{0}}^{t_{0}+T} \mathcal{D}_{i}(t) d t .
$$

Here, $t_{0}$ and $T$ are characteristic times of the flow. In practice, in our simulations the pointwise turbulent diffusion coefficients $\mathcal{D}_{i}\left(x_{i}, t\right)$ first grow in time, as the initial Gaussian profile is mixed by the turbulence, reach a maximum, and then decrease as the scalar becomes diluted (which happens in most runs after at most three turnover times). This is reminiscent of the behavior of the energy dissipation in freely decaying turbulence, which reaches a maximum and then decreases. Following a standard practice when studying decaying flows, in the runs we chose $t_{0}$ as the time of saturation in the growth of $\mathcal{D}_{i}(t)$, and $T \approx 1$, proportional to the turnover time. 


\section{NUMERICAL RESULTS}

\section{A. Isotropic turbulence}

In the absence of rotation (runs in set A), we expect the diffusion coefficients to be isotropic (i.e., the horizontal and vertical diffusions to be the same within error bars). Figure 1 shows the horizontal average profile of the passive scalar $\bar{\theta}(x, t)$, the horizontal flux $\overline{\theta u_{x}}(x, t)$, and the pointwise horizontal diffusion $\mathcal{D}_{x}(x, t)$ at five different times in run $A_{x 1}$. As time evolves, the mean profile $\bar{\theta}(x, t)$ flattens and widens. The flux is roughly antisymmetric and is positive for $x>\pi$ and negative for $x<\pi$. This can be expected as there is an excess of $\theta$ at $x=\pi$ at $t=0$ which turbulent mixing should diffuse, by transporting this excess towards $x=0$ and $2 \pi$. Horizontal diffusion increases to its saturation value around $t_{0} \approx 1.5$; after this time, it fluctuates around a mean value. Large fluctuations in $\mathcal{D}_{x}(x, t)$ near the center of the box are due to the fact that, by definition, turbulent diffusion diverges at that point.
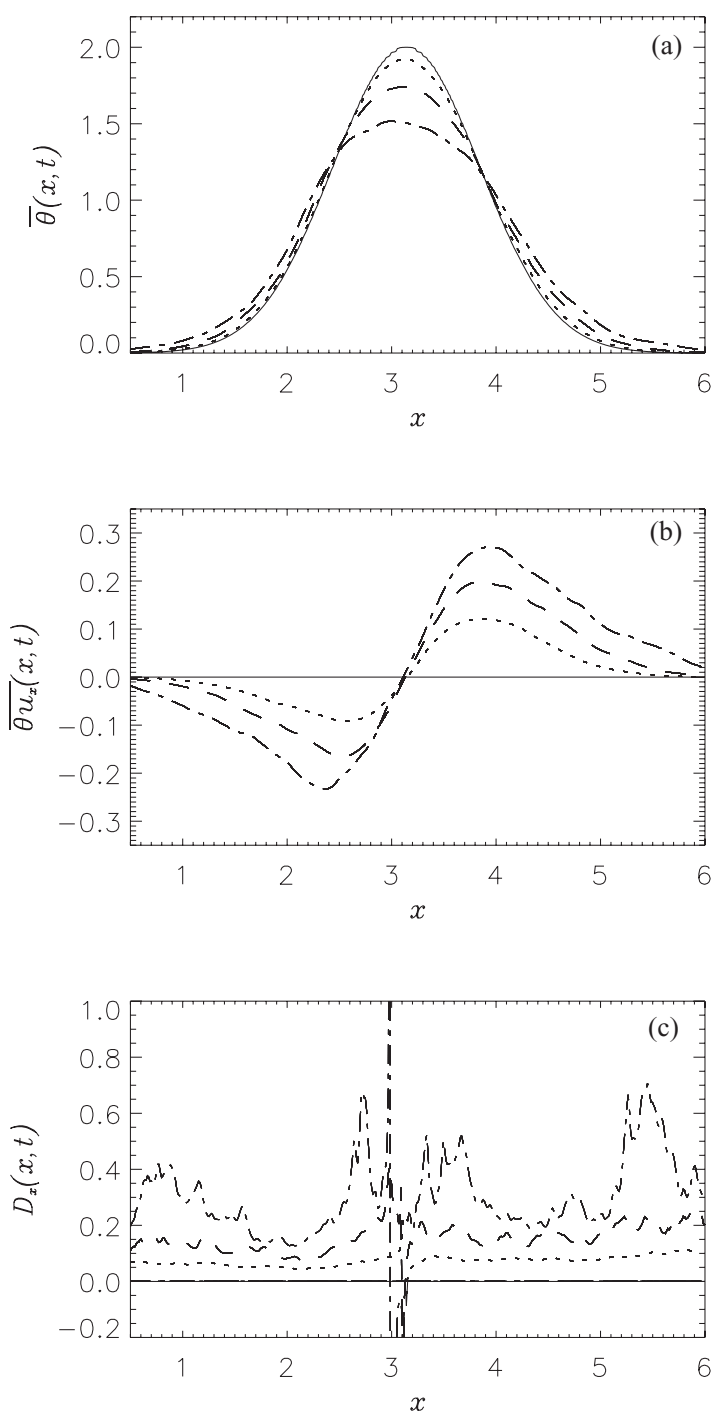

FIG. 1. (a) Average horizontal concentration $\bar{\theta}(x, t)$ in run $A_{x 1}$, at times $t=0,0.5,1$, and 1.5, denoted by solid, dotted, dashed, and dashed-dotted lines respectively. (b) Horizontal flux at the same times. (c) Pointwise horizontal turbulent diffusion at the same times.
Passive scalar concentration, vertical flux, and vertical turbulent diffusion were also calculated for run $A_{z 1}$ (i.e., the same run but with an initial Gaussian profile in $z$ ). As expected, the same results as in run $A_{x 1}$ were obtained.

\section{B. Effect of rotation}

\section{Horizontal transport}

Figure 2 shows the mean horizontal concentration $\bar{\theta}(x, t)$, the horizontal flux $\overline{\theta u_{x}}(x, t)$, and the pointwise horizontal diffusion $\mathcal{D}_{x}(x, t)$ for different times in run $C_{x 1}(\Omega=4)$. In this case, diffusion grows faster at earlier times, but then saturates at a lower value. Also note that the average profile and the average flux become asymmetric: there is an excess of $\bar{\theta}(x, t)$ for $x<\pi$, and the flux (in absolute value) is larger for $x<\pi$ than for $x>\pi$. This asymmetry is associated with the Coriolis force and has been already observed in [8]. As will be shown
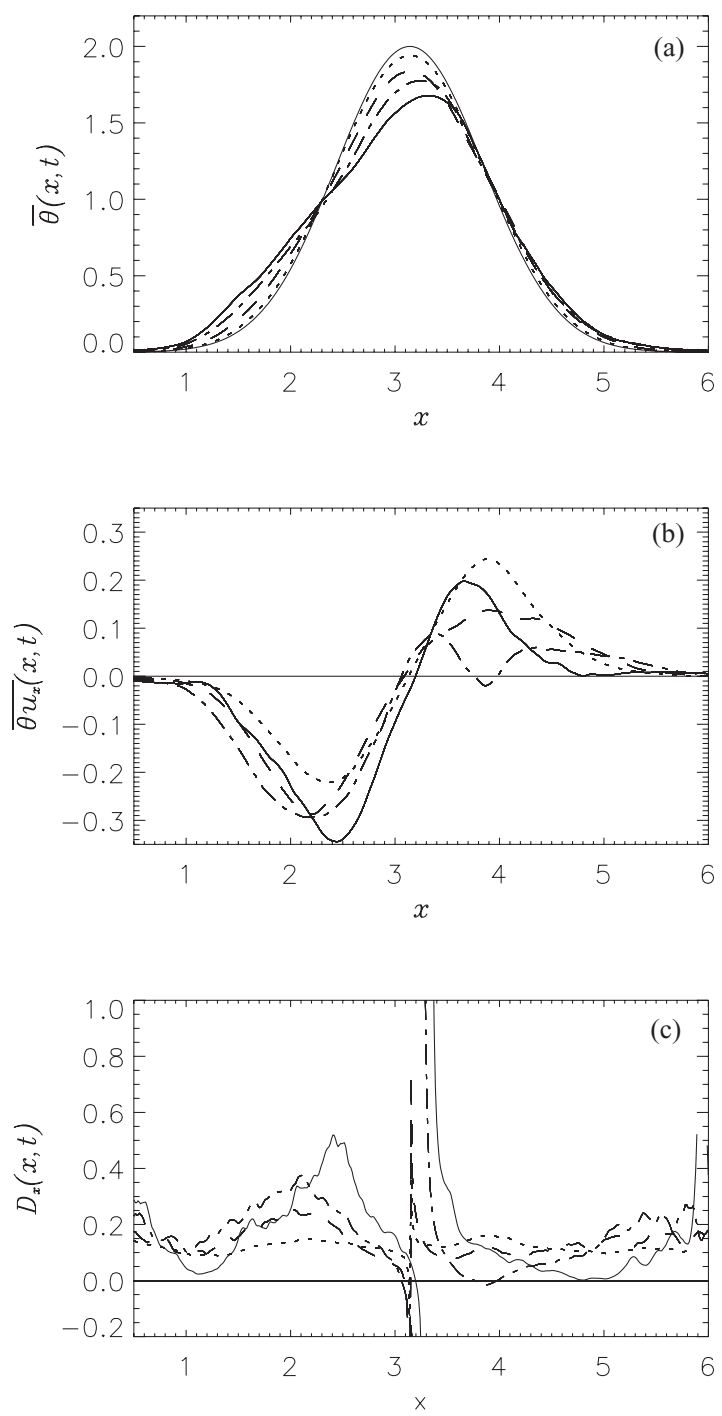

FIG. 2. (a) Averaged vertical concentration $\bar{\theta}(x, t)$ in run $C_{x 1}$, at times $t=0,0.25,0.5,0.75$, and 1 , denoted by solid, dotted, dashed, dashed-dotted, and dashed-triple-dotted lines, respectively. (b) Horizontal flux at the same times. (c) Pointwise horizontal turbulent diffusion at the same times. 

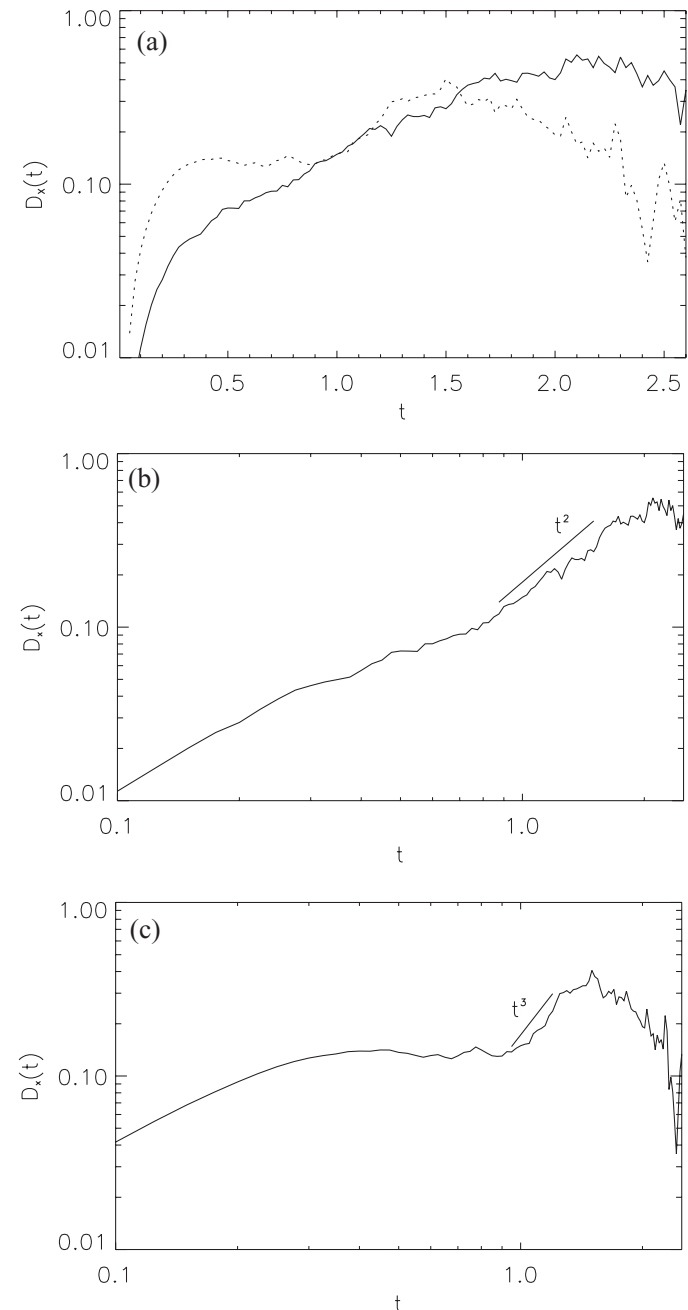

FIG. 3. (a) Horizontal turbulent diffusion as a function of time for runs $A_{x 1}$ (solid line) and $C_{x 1}$ (dotted line). (b) Detail in log-log coordinates of the horizontal turbulent diffusion for run $A_{x 1}$. (c) Same for run $C_{x 1}$.

later, it results from a rotation of the initial passive scalar profile.

From $\mathcal{D}_{x}(x, t)$ calculated for each time, we obtain the turbulent diffusion coefficient $\mathcal{D}_{x}(t)$ by computing its mean over all values of $x$. Figure 3 shows the resulting horizontal turbulent diffusion as a function of time for runs $A_{x 1}$ and $C_{x 1}$. In both runs, $\mathcal{D}_{x}(t)$ grows from an initially small value to a saturation value around $t_{0} \approx 1.5$ in run $A_{x 1}$ and $t_{0} \approx 1.2$ in run $C_{x 1}$. These two values are typical of all runs without and with rotation, respectively, and were used to compute the time average of $\mathcal{D}_{x}(t)$ using Eq. (11). Note that $t_{0}$ is smaller in run $C_{x 1}$, as the runs with rotation show a faster growth of $\mathcal{D}_{x}(t)$ at early times, but saturate faster at a lower value. Later, $\mathcal{D}_{x}(t)$ decreases as the passive scalar is diffused and homogeneity is finally recovered. As can be seen in Fig. 3(a), this decrease is more evident in run $C_{x 1}$.

As already mentioned, although at early times $\mathcal{D}_{x}(t)$ grows faster in the run with rotation (run $\left.C_{x 1}\right), \mathcal{D}_{x}(t)$ saturates at a lower value than in run $A_{x 1}$. This indicates horizontal diffusion decreases in the presence of rotation, in comparison with the isotropic and homogeneous cases. This is in good agreement with predicted reductions of the horizontal passive scalar transport in the presence of rotation [22,23], and also with previous measurements of turbulent transport coefficients in numerical simulations using a different method (the test field model, see [8]).

It is interesting that at intermediate times the growth of $\mathcal{D}_{x}(t)$ can be roughly explained by a simple phenomenological model. If we think that turbulent eddies dominate the transport over molecular diffusion, and we think of the turbulent flow as a superposition of eddies at different scales $\ell$, then we can imagine that the smallest eddies (much smaller than the integral scale eddies), with faster turnover times $\tau_{\ell}$, are the first to start mixing the passive scalar. As time evolves, larger and larger eddies come into play, as the eddies at larger scales are able to complete a turnover. For eddies in the inertial range, the eddy turnover time can be estimated as

$$
\tau_{\ell} \sim \ell / u_{\ell}
$$

In isotropic and homogeneous turbulence, the inertial range scaling for the velocity field (in the absence of intermittency corrections) can be written as $u_{\ell} \sim \ell^{1 / 3}$. Then, the eddy turnover time is

$$
\tau_{\ell} \sim \ell^{2 / 3}
$$

From mixing length arguments, the turbulent diffusion at late times is $\mathcal{D}_{x} \sim L U$. At early times, if only the eddies with turnover time smaller than the actual time contribute to the mixing, then from Eqs. (12) and (13) we get

$$
\mathcal{D}_{x} \sim \tau_{\ell}^{2} \text {. }
$$

In the rotating case the inertial range scaling for the velocity field is modified. Rotation sets a preferential direction for energy transfer, resulting in a quasibidimensionalization of the flow. Assuming $E\left(k_{\perp}\right) \sim k_{\perp}^{-2}$ in the inertial range (see, e.g., [33,34]), it follows that $u_{\ell_{\perp}} \sim \ell_{\perp}^{1 / 2}$. If at late times we can assume the horizontal diffusion $\mathcal{D}_{x} \sim L_{\perp} U_{\perp}$ (with $L_{\perp}$ and $U_{\perp}$, respectively, the characteristic length and velocity in the perpendicular direction), and using the same arguments as in the isotropic and homogeneous cases, then

$$
\mathcal{D}_{x} \sim \tau_{\ell}^{3} .
$$

Assuming that $t \sim \tau_{\ell}$ (i.e., that at a given time only the eddies that were able to do a full turn contribute to the mixing), one could expect to observe the growth given by Eqs. (14) and (15) before saturation in $\mathcal{D}_{x}(t)$. These scaling laws are indicated as a reference in Figs. 3(b) and 3(c). Although there is an agreement with the prediction, the ranges are shorter than a decade and we can not conclude that the scalings appear in the simulations. Also, the behavior of run $A_{x 1}$ at early times can be also compatible with exponential growth of $\mathcal{D}_{x}(t)$. Given the large fluctuations in $\mathcal{D}_{x}(t)$ in all runs, more simulations with the same parameters (but with different initial conditions to perform an ensemble average) would be needed to study this early time behavior in more detail.

The reduction of the saturation value of the horizontal turbulent diffusion observed in Figs. 1, 2, and 3 can be further confirmed by studying the mean temporal value of $\mathcal{D}_{x}(t)$ (namely, $\mathcal{D}_{x}$ ) for all runs with $S_{c}=1$. Figure 4 shows the mean temporal value of the horizontal diffusion as a function 


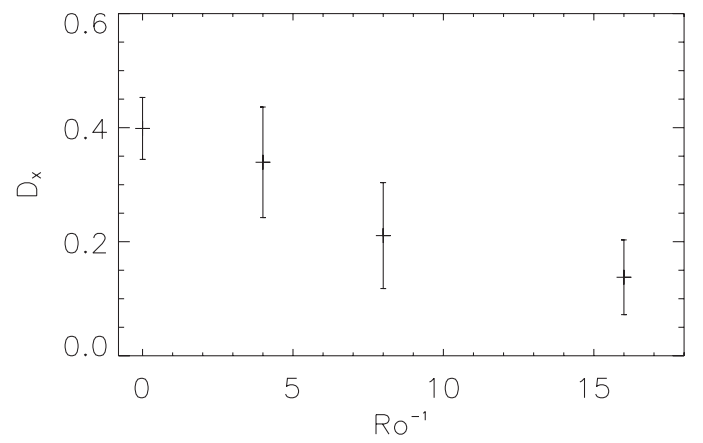

FIG. 4. Mean horizontal turbulent diffusion as a function of the inverse Rossby number, obtained from simulations $A_{x 1}, B_{x 1}, C_{x 1}$, and $E_{x 1}$ (all runs with $S_{c}=1$ ). Error bars correspond to the mean standard deviation.

of the Rossby number for runs $A_{x 1}, B_{x 1}, C_{x 1}$, and $E_{x 1}$ (i.e., simulations with $\Omega=0,2,4$ and 8 , and with initial Gaussian profile of the scalar in $x$ ). The error bars correspond to the mean standard deviation. It is clear from the data that $\mathcal{D}_{x}$ decreases monotonically as $1 / R_{o}$ increases.

\section{Vertical diffusion}

Figure 5 shows the mean vertical passive scalar concentration $\bar{\theta}(z, t)$, the mean vertical flux $\overline{\theta v_{z}}(z, t)$, and the pointwise vertical diffusion $\mathcal{D}_{z}(z, t)$ at different times in run $C_{z 1}$. Note the profiles here are more similar to those obtained in the isotropic and homogeneous cases: $\bar{\theta}(z, t)$ and $\overline{\theta v_{z}}(z, t)$ are, respectively, symmetric and antisymmetric with respect to $z=\pi$.

As in the case of horizontal diffusion, we can compute the mean vertical diffusion coefficient in runs $A_{z 1}, B_{z 1}, C_{z 1}$, and $E_{z 1}$. This results in a dependence of $\mathcal{D}_{z}$ with the Rossby number, and is shown in Fig. 6. While mean horizontal diffusion is strongly dependent on the Rossby number, the mean vertical diffusion seems to be (within error bars) independent of its value.

\section{Effect of $S_{c}$ and $P_{e}$ numbers}

A large number of runs were performed to study the effect of the Schmidt number in turbulent diffusion. As the molecular diffusivity $\kappa$ is increased, we can expect turbulent diffusion to decrease until molecular diffusion dominates. Figure 7 shows the mean turbulent diffusion $\mathcal{D}_{x}$ as a function of $P_{e}$ in runs with $\Omega=0$ (i.e., estimating $\mathcal{D}_{x}$ from all the runs in set $A$ ). As the flow is approximately isotropic, $\mathcal{D}_{x} \approx \mathcal{D}_{z}$.

Effects associated with the Pèclet and Schmidt numbers can be observed for small $P_{e}$, as $\mathcal{D}_{x}$ starts to decrease for $P_{e} \lesssim 100$. However, for a wide range of values of the Pèclet number, $\mathcal{D}_{x}$ remains approximately constant. This is in good agreement with theoretical expectations that for small enough $\nu$ and $\kappa$, the effective turbulent Schmidt number should be of order one (see [35] for similar arguments in the case of the turbulent magnetic Prandtl number).

A similar behavior is observed in the rotating case, as can be seen from Figs. 8 and 9. In these figures, the turbulent transport coefficients are computed from the runs in set $C(\Omega=4$, $R_{o}=0.02$ ). As in the isotropic case, for sufficiently large $P_{e}$, turbulent diffusion remains approximately constant and the
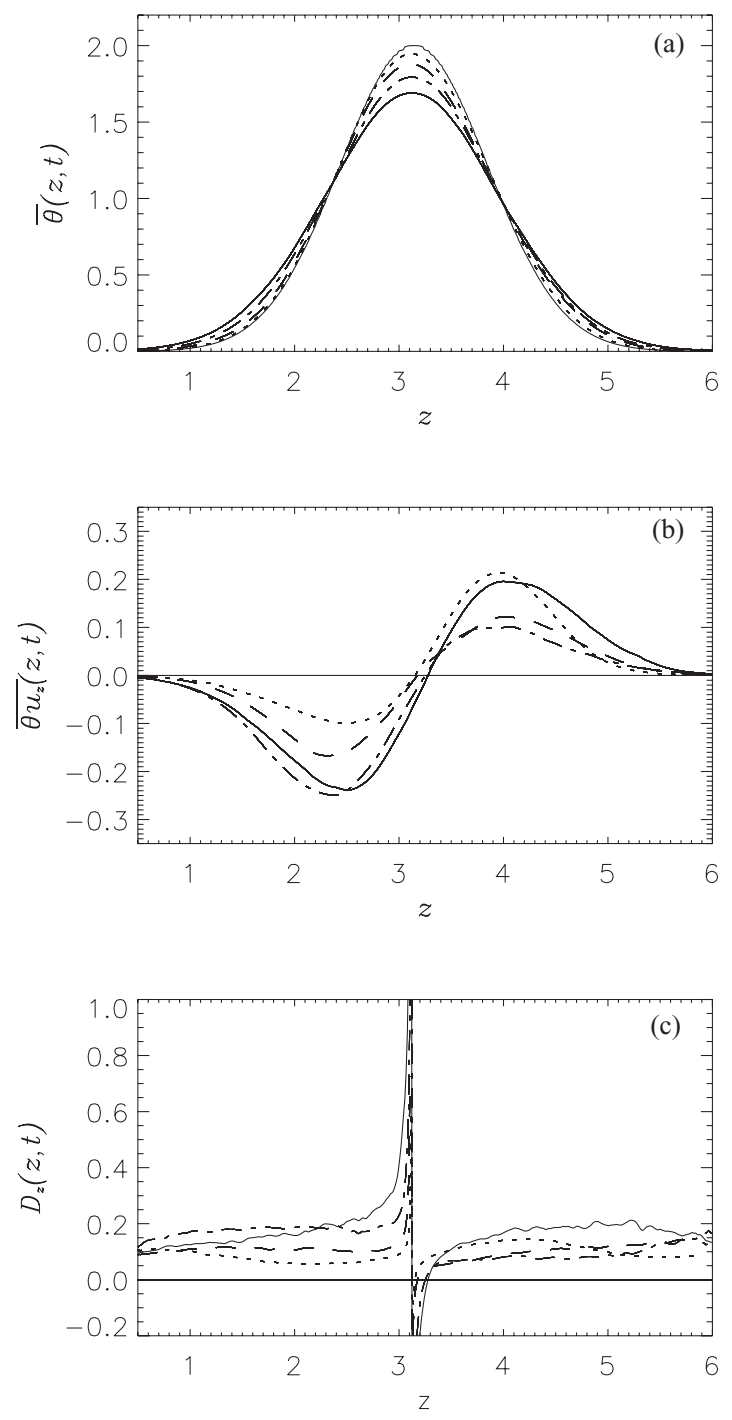

FIG. 5. (a) Averaged passive scalar profile $\bar{\theta}(z, t)$ in run $C_{z 1}$, at times $t=0,0.25,0.5,0.75$, and 1 , denoted by solid, dotted, dashed, dashed-dotted, and dashed-triple-dotted lines, respectively. (b) Mean vertical flux as a function of $z$ at different times in the same run. (c) Same for the pointwise vertical turbulent diffusion.

effective Schmidt number is of order one. $P_{e}$ number effects are observed earlier for vertical diffusion at small $P_{e}$ (indeed, for

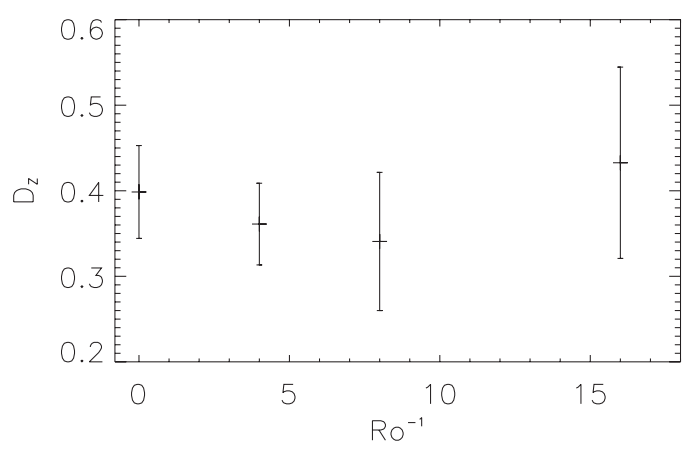

FIG. 6. Mean vertical turbulent diffusion as a function of the inverse Rossby number, obtained from simulations $A_{z 1}, B_{z 1}, C_{z 1}$, and $E_{z 1}$ (all runs with $S_{c}=1$ ). Error bars correspond to the mean standard deviation. 


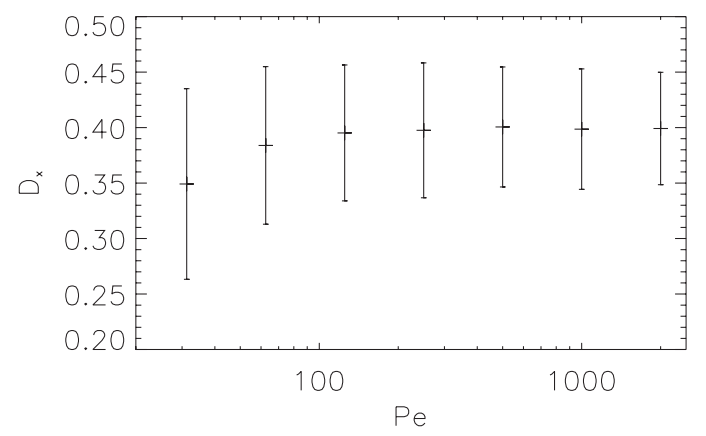

FIG. 7. Mean turbulent diffusion $\mathcal{D}_{x}$ as a function of the Pèclet number for runs $A_{x 1}$ to $A_{x 32}(\Omega=0)$. Note that as the flow in these runs is approximately isotropic, $\mathcal{D}_{x} \approx \mathcal{D}_{z}$.

$P_{e} \leqslant 500$ a change in the behavior of $\mathcal{D}_{z}$ can already be seen), although it seems that much smaller $P_{e}$ numbers are required to see a significant decrease in the horizontal diffusion. This could be associated with the fact that in the presence of rotation the turbulent diffusivity is already reduced, and that therefore larger molecular diffusivities are needed to decrease it further. Also, note that in the runs with large enough $P_{e}$ and small enough $R_{o}$, the vertical turbulent diffusion reaches a value that is approximately twice the horizontal, in good agreement with predictions in $[19,22]$.

The change in the turbulent diffusivity as the $P_{e}$ number is decreased can be also understood from the spectra of energy and passive scalar variance (see Fig. 10). For $S_{c}=1$, at late times the energy and the passive scalar variance display an inertial range in a similar range of wave numbers. As a result, it can be expected that the turbulent eddies will mix the passive scalar concentration at all scales. For small $S_{c}$ and $P_{e}$, the passive scalar can not develop an inertial range. As a result, eddies smaller than the dissipation scale for the passive scalar (i.e., eddies in the inertial range of the velocity field) are unable to mix the passive scalar and thus the turbulent diffusion decreases.

\section{Turbulent structures and diffusion}

From the results presented above, it is clear that rotation plays an important role in the diffusion of the passive scalar, modifying its mixing with respect to the isotropic and homogeneous cases. Figure 11 shows a cut in a horizontal

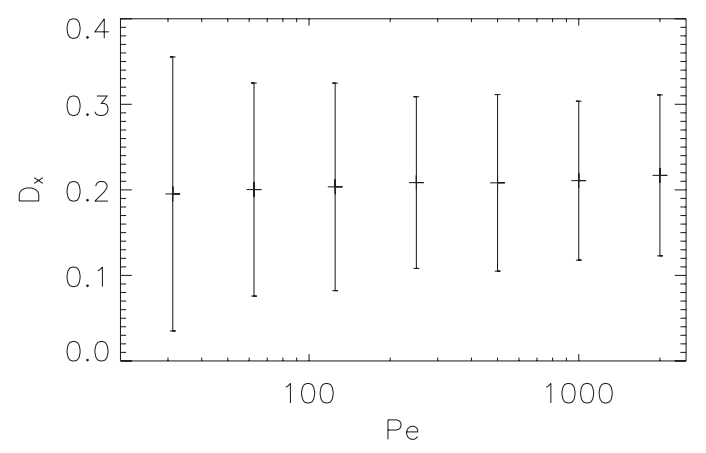

FIG. 8. Mean horizontal turbulent diffusion as a function of the Pèclet number for runs $C_{x 1}$ to $C_{x 32}(\Omega=4)$.

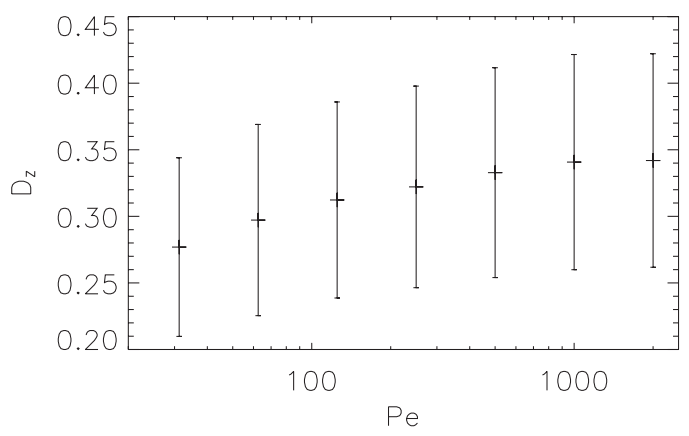

FIG. 9. Mean vertical trubulent diffusion as a function of the Pèclet number for runs $C_{z 1}$ to $C_{z 32}(\Omega=4)$.

plane of the passive scalar concentration in run $C_{x 1}$ at different times. The initial Gaussian profile not only diffuses in time, but also bends and rotates. The bending of the passive scalar concentration was observed before in [8] and explained as an effect of the Coriolis force. In our runs, the passive scalar at $t=0$ is concentrated in a narrow band around $x=\pi$. The average flux is thus towards positive values of $x$ for $x>\pi$, and towards negative values of $x$ for $x<\pi$ (i.e., in the direction of $-\nabla \theta$, see Fig. 2). The Coriolis force in Eq. (1) is $-2 \Omega \hat{z} \times \mathbf{u}$ and therefore on the average this force creates a drift of the flux towards positive values of $y$ in the $x>\pi$ region, and towards negative values of $y$ for $x<\pi$ [8]. This explains the bending of the initial profile we observe of the runs with rotation, which is not observed in the runs without rotation (see Fig. 12 for a comparison).

Diffusion in the parallel direction is of a different nature, and more strongly dependent of the structures that emerge
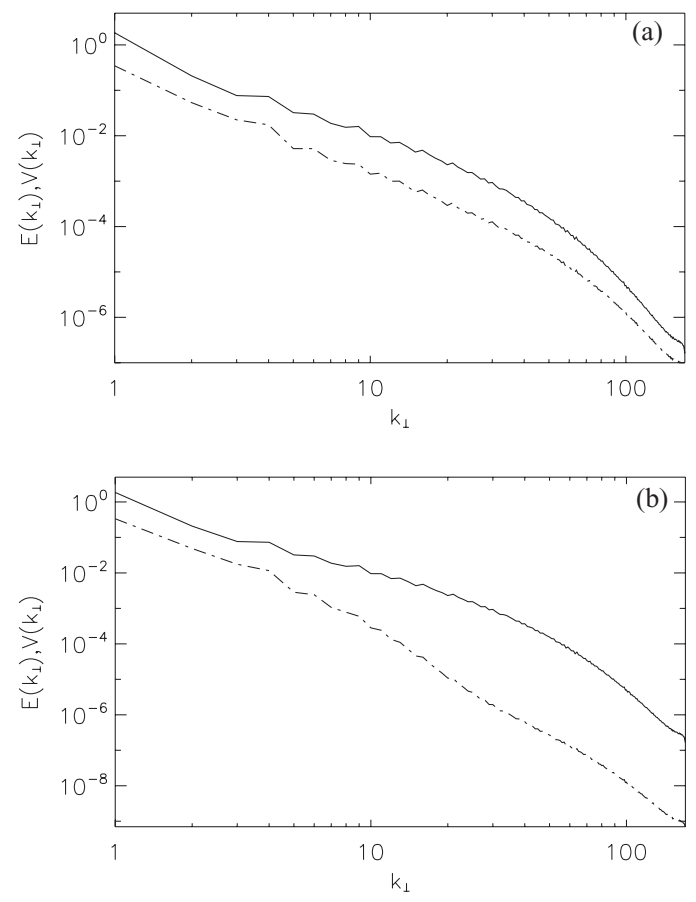

FIG. 10. (a) Horizontal energy spectrum (solid line) and passive scalar variance spectrum (dashed-dotted line) at late times in run $C_{x 1}$. (b) Same for run $C_{x 32}$. 

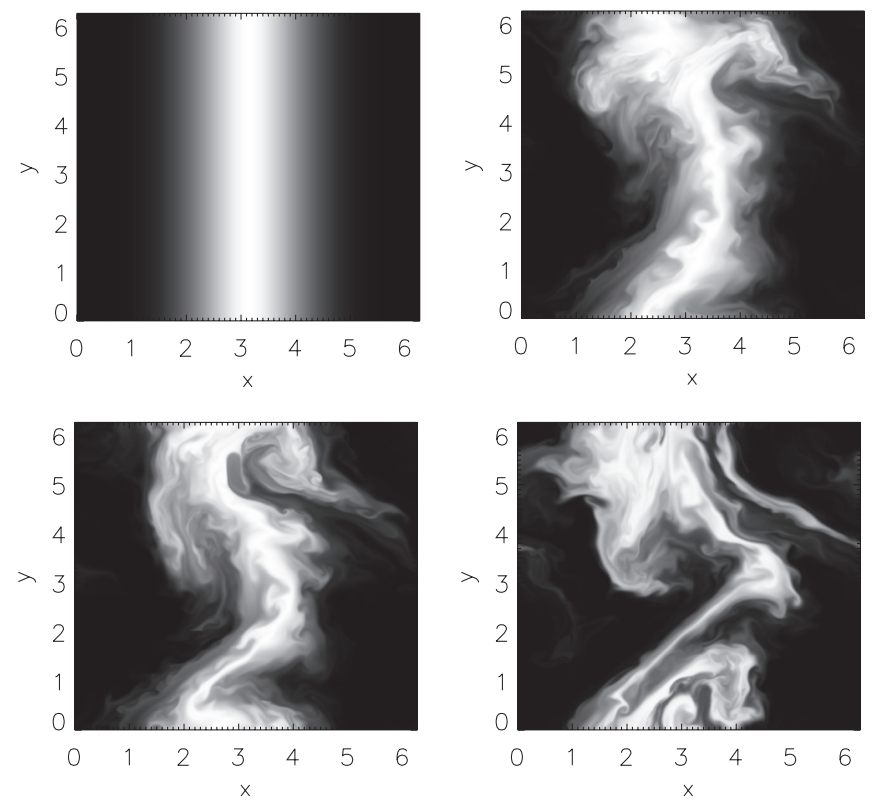

FIG. 11. Passive scalar concentration in a horizontal plane in run $C_{x 1}$, at times $t=0,1,1.5$, and 2.5 from left to right and from top to bottom.

in rotating turbulent flows. Rapidly rotating flows are characterized by columnar structures in the velocity field and vorticity, associated with a quasibidimensionalization of the flow. The mechanism underlying the transfer of energy towards two-dimensional modes and responsible for the formation of these columns seems to be associated with wave resonances in the energy-exchanging triadic interactions [36]. Two-point closures of turbulence, such as the eddy damped quasinormal Markovian closure (see, e.g., [33]) successfully explain the emergence of columns with the same principle. However, there are alternative theories that consider the formation of columns as the result of a relative concentration of kinetic energy in cylindrical structures resulting from the conservation of linear and angular momentum [37].

Columnar structures have been reported in many numerical simulations of turbulent flows (see, e.g., [38]). As these columns live for long times and move across the domain, they play an important role in the mixing of the passive scalar. Figure 13 shows a cut in a vertical plane of the passive scalar concentration at different times in run $C_{z 1}$. Note that diffusion is different from the one observed in horizontal planes in the
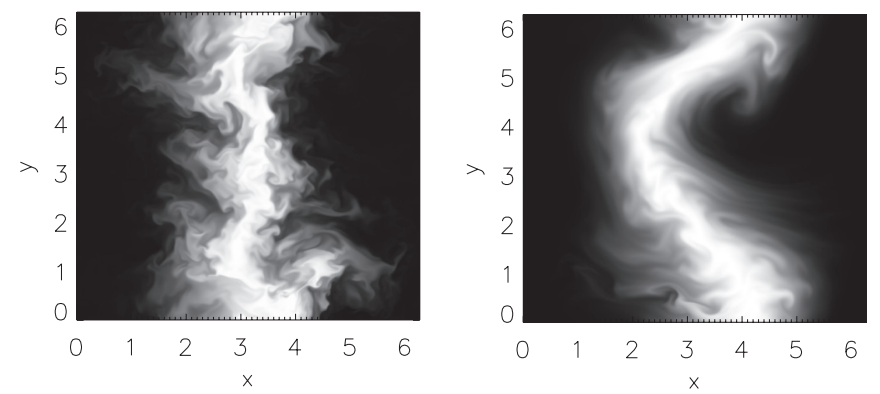

FIG. 12. Passive scalar concentration in a horizontal plane at $t=1$ in runs $A_{x 1}$ (left, no rotation) and $E_{x 1}$ (right, $\Omega=8$ ).
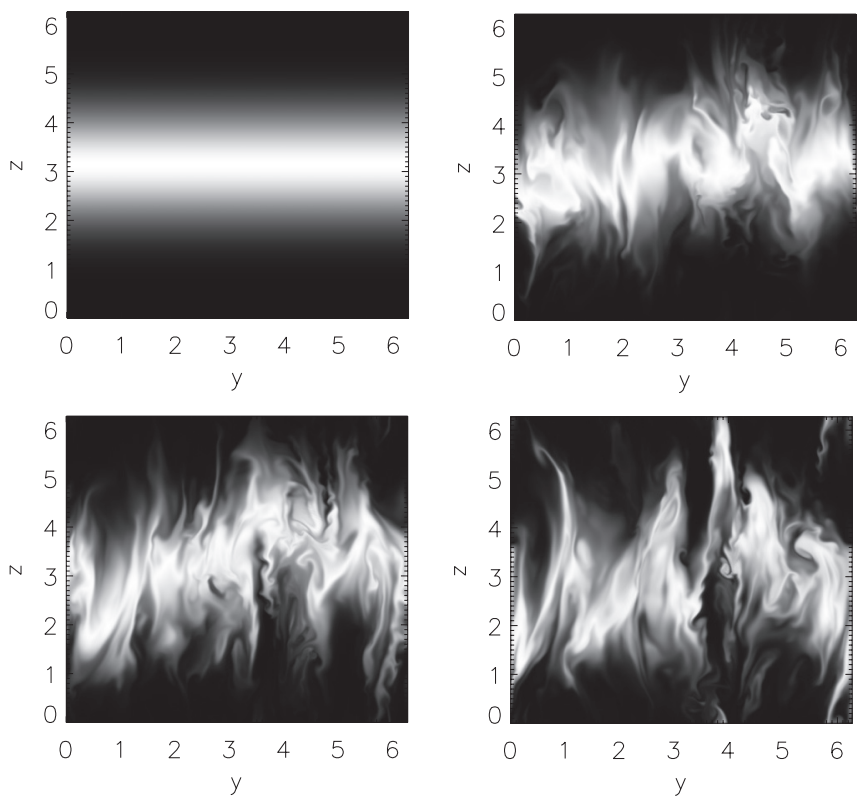

FIG. 13. Passive scalar concentration in a vertical plane in run $C_{z 1}$, at times $t=0,1,1.5$, and 2.5 from left to right and from top to bottom.

same run (Fig. 11), and from the one observed in the isotropic and homogeneous case [Fig. 12(a)]. The passive scalar is diffused from its initial profile in vertical stripes, which are stretched further (thus increasing the mixing) as time evolves. These stripes are created by updrafts or downdrafts inside the columns. As these columns go through the region with large concentration of the passive scalar, the updrafts or downdrafts mix the passive scalar with the regions immediately above or below.

\section{CONCLUSIONS}

We used 56 direct numerical simulations with regular spatial resolution of $512^{3}$ grid points to measure turbulent diffusion in directions parallel and perpendicular to the rotation axis, in turbulent flows at different Rossby and Schmidt numbers. The effective coefficients were obtained by studying the diffusion of an initial concentration of the passive scalar and calculated by measuring its average concentration and average spatial flux.

The effect of rotation in turbulent diffusion is the opposite of that found in the presence of stratification [7,23]: while in the latter case stratification reduces vertical diffusion with respect to horizontal diffusion, rotation dramatically reduces scalar diffusivity in the horizontal direction. In our simulations, vertical diffusion remains of the same order as in isotropic and homogeneous turbulence, although for a different reason: in rotating flows, it was found that diffusion in the vertical direction is strongly dominated by updrafts and downdrafts in columnar structures in the velocity field.

Within error bars, and for small enough Rossby number and large enough Schmidt number, our results are consistent with theoretical results based on single-particle dispersion that predict that vertical diffusion is twice larger than horizontal 
diffusion in the presence of pure rotation [19,22]. For small Schmidt and Pèclet numbers, the turbulent diffusion decreases as molecular diffusion becomes more important, while for large enough Pèclet numbers the effective diffusion becomes independent of the Pèclet number, resulting in a turbulent Schmidt number of order one.

\section{ACKNOWLEDGMENTS}

The authors acknowledge support from Grants No. PIP 11220090100825, No. UBACYT 20020110200359, and No. PICT 2011-1529 and No. 2011-1626. P.D.M. acknowledges support from the Carrera del Investigador Científico of CONICET.
[1] E. Schatzman, Astron. Astrophys. 56, 211 (1977).

[2] C. Charbonnel, S. Vauclair, and J. P-Zahn, Astron. Astrophys. 255, 191 (1992).

[3] G. Rüdiger and V. V. Pipin, Astron. Astrophys. 375, 149 (2001).

[4] P. H. Roberts and G. A. Glatzmaier, Rev. Mod. Phys. 72, 1081 (2000).

[5] R. Rotunno and G. H. Bryan, J. Atmos. Sci. 69, 2284 (2012).

[6] T. R. Osborn, J. Phys. Oceanogr. 10, 83 (1980).

[7] A. Vincent, G. Michaud, and M. Meneguzzi, Phys. Fluids 8, 1312 (1996).

[8] A. Brandenbourg, A. Svedin, and G. M. Vasil, Mon. Not. R. Astron. Soc. 395, 1599 (2009).

[9] Z. Warhaft, Annu. Rev. Fluid Mech. 32, 203 (2000).

[10] G. Falkovich, K. Gawedzki, and M. Vergassola, Rev. Mod. Phys. 73, 913 (2001).

[11] A. Celani, M. Cencini, A. Mazzino, and M. Vergassola, New J. Phys. 9, 1367 (2004).

[12] V. V. Pipin, Astron. Lett. 27, 203 (2001).

[13] G. Bryan and R. Rotunno, J. Atmos. Sci. 66, 3042 (2009).

[14] G. Bryan and H. Morrison, Mon. Weather Rev. 140, 202 (2012).

[15] L. Liechtenstein, F. S. Godeferd, and C. Cambon, J. Turbul. 6, N24 (2005).

[16] P. Rodriguez Imazio and P. D. Mininni, Phys. Rev. E 83, 066309 (2011).

[17] R. H. Kraichnan, Phys. Rev. Lett. 72, 1016 (1994).

[18] F. Nicolleau and J. C. Vassilicos, J. Fluid Mech. 410, 170 (2000).

[19] C. Cambon, F. S. Godeferd, F. C. G. A. Nicolleau, and J. C. Vassilicos, J. Fluid Mech. 499, 231 (2004).
[20] Y. Kaneda and T. Ishida, J. Fluid Mech. 402, 311 (2000).

[21] Y. Kaneda, J. Phys. Soc. Jpn. 69, 3847 (2000).

[22] F. Nicolleau, G. Yu, and J. C. Vassilicos, Fluid Dyn. Res. 40, 68 (2008).

[23] Y. Kimura and J. R. Herring, J. Fluid Mech. 328, 253 (1996).

[24] R. E. Britter, J. C. R. Hunt, G. L. Marsh, and W. H. Snyder, J. Fluid Mech. 127, 27 (1983).

[25] L. Del Castello and H. J. H. Clercx, Phys. Rev. E 83, 056316 (2011).

[26] P. K. Yeung and J. Xu, Phys. Fluids 16, 93 (2004).

[27] G. H. Bryan and R. Rotunno, Mon. Weather Rev. 137, 1770 (2009).

[28] G. H. Bryan, Mon. Weather Rev. 140, 1125 (2012).

[29] S. A. Hausman, Ph.D. dissertation, Colorado State University.

[30] J. Persing and M. T. Montgomery, J. Atmos. Sci. 60, 2349 (2003).

[31] D. O. Gómez, P. D. Mininni, and P. Dmitruk, Adv. Space Res. 35, 899 (2005).

[32] D. O. Gómez, P. D. Mininni, and P. Dmitruk, Phys. Scr. T116, 123 (2005).

[33] C. Cambon and L. Jacquin, J. Fluid Mech. 202, 295 (1989).

[34] P. D. Mininni and A. Pouquet, Phys. Rev. E 79, 026304 (2009).

[35] Y. Ponty, P. D. Mininni, D. C. Montgomery, J. F. Pinton, H. Politano, and A. Pouquet, Phys. Rev. Lett. 94, 164502 (2005)

[36] F. Waleffe, Phys. Fluids A 4, 350 (1992).

[37] P. A. Davidson, P. J. Staplehurst, and S. B. Dalziel, J. Fluid Mech. 557, 135 (2006).

[38] P. D. Mininni, A. Alexakis, and A. Pouquet, Phys. Fluids 21, 015108 (2009). 